\title{
Zeno and anti-Zeno effect in an open quantum system in the ultrastrong-coupling regime
}

\author{
Shu $\mathrm{He}^{1}$, Qing-Hu Chen ${ }^{1,2, *}$, and Hang Zheng ${ }^{3}$ \\ 1 Department of Physics, Zhejiang University, Hangzhou 310027, China \\ 2 Collaborative Innovation Center of Advanced Microstructures, Nanjing 210093, China \\ 3 Department of Physics, Shanghai Jiao Tong University, Shanghai 200240, China
}

(Dated: June 12, 2017)

\begin{abstract}
We study the quantum Zeno effect (QZE) and quantum anti-Zeno effect (QAZE) of a two-level system interacting with an environment of harmonic oscillators, the spin-boson model. By applying a numerically exact method based on matrix product states, the previously obtained picture on the influence of counter-rotating terms has to be modified: For physical bath initial states, the transition from QZE to QAZE with increasing measurement interval is only absent at weak coupling, while present at strong coupling. Furthermore, we find that the transition occurs always for the widely used bare bath initial state. Within a more realistic measurement scheme where only the qubit is projectively measured, the above scenario for the bare bath initial state remains qualitatively unchanged, apart from accelerated decay for intermediate measurement intervals.
\end{abstract}

PACS numbers: 03.65.Ge, 03.65.Xp, 42.50.Pq

\section{INTRODUCTION}

Frequent measurements of a quantum system may slow down its dynamic evolution. This phenomenon is known as the quantum Zeno effect (QZE) [1]. On the other hand, the decay rate could also be accelerated by frequent measurements under somewhat different conditions. This opposite effect is generally called the quantum anti-Zeno effect (QAZE) [2]. The change in the evolution implies a potential strategy to control the quantum dynamics of a target system. It can be used to protect quantum information [3], suppress decoherence [4] and even cool down and purify a quantum system [5]. Experimentally, both QZE and QAZE were investigated in various contexts such as trapped ions [6], ultracold atoms [7], nanomechanical oscillators [8], superconducting circuits [9], and cavity quantum electrodynamics systems [10]. One of the important concepts employed for quantum control is the QZE-QAZE transition. It occurs in a qubit interacting with its environment where repetitive measurements are performed which project the whole system (qubit plus environment) to its initial state. Recently, the ultra-strong coupling regime between qubit and environment has been realized within cavity quantum electrodynamics and superconducting circuits [11-13]. In this case, the conventional rotating-wave approximation (RWA), the standard in quantum optics, is no longer valid. It is therefore mandatory to go beyond it also in the study of the Zeno effects.

It is well known that both QZE and QAZE always happen within an RWA-based approach [1, 18]. However, recent studies going beyond the RWA have shown that the counter-rotating terms play a significant role for both QZE and QAZE [15 17], although they were only partially taken into account. Zheng et al. found that there is no QAZE for hydrogen [15] if the decay from a physical excited initial state is computed including counterrotating terms to the second order. Ai et al. studied systematically the spontaneous decay phenomenon of a two-level system under the influence of both the environment and repetitive measurements without the RWA [17], and found that the QAZE can still happen in some cases ( even without the RWA ) if the initial state is a bare excited state. For a physically excited state, they also observed that the QAZE disappears and the QZE is always robust.

For a reliable study of the Zeno effects in quantum dissipative systems, the exact dynamics is needed in principle. Actually, the dynamics of quantum open systems has been studied for a long time using a variety of methods; an incomplete list is given by the authors of Refs. [1931]. For a Lorentzian spectral function, it is generally believed that the numerically exact hierarchical equationsof-motion approach can be applied. Exact dynamics for more general baths, such as Ohmic and sub-Ohmic baths, were claimed by several groups [22, 30, 31]. For the Zeno effects, the other crucial ingredient is the measurement scheme. In most experimentally realizable processes, measurements are applied on the system (qubit) only and the state of the environment after each measurement will deviate from its initial state [33]. This applies certainly in the ultra-strong coupling regime due to the strong correlation between the system and its environment. The ideal assumption that the measurement projects the state of the system together with the environment to the total initial state breaks down. Recently, the effect induced by partial measurement was considered in the dephasing process based on an exactly solvable model 34. Therefore it is very important to see how this more realistic measurement scheme affects the QZE-QAZE transition of the dissipative open quantum system in the ultra-strong coupling regime.

In this paper, we study the QZE and QAZE of an open quantum system where a two-level system interacts with its environment for both Ohmic and sub-Ohmic baths, the paradigmatic spin-boson model. We focus mainly on 
the ultra-strong coupling regime where both the RWA and the ideal measurement assumption are not valid. We employ a highly efficient and numerically exact method based on a recently proposed time-dependent variational principle (TDVP) for matrix product states (MPS) [35], improved in [31, 32], to study the evolution from several initial states. It was shown that quantum fluctuations of the environment manifested in a huge number of environmental bosons generated during time evolution can be precisely described within this approach 31]. We compare our result of the QZE and QAZE transitions with previous studies based on a conventional unitary transformation (UT) approach, where the effect of counterrotating terms is not fully taken into account [15, 17]. By TDVP, we also study the QZE and QAZE within the more realistic measurement scheme described above, where only the qubit is projected onto the initial state while the environment continues to evolve [36].

The paper is organized as follows. In Sec. II, we briefly introduce the TDVP approach as applied to the open quantum system. Then we employ it to study the spin dynamics, and compare to the results obtained by the UT approach in Sec. III. In Sec. IV, we use the numerical exact TDVP method to study the QZE. The results for two initial states are given, and comparison to previous results by the UT approach is performed. Furthermore, the TDVP method is used to study the QZE and QAZE within a more realistic measurement scheme. We close with a short summary in Sec. V.

\section{TIME-DEPENDENT VARIATIONAL PRINCIPLE}

A qubit coupled to its environment of harmonic oscillators can be described by the following spin-boson Hamiltonian $(\hbar=1)$ :

$$
H=\frac{1}{2} \Delta \sigma_{z}+\frac{1}{2} \sum_{k} g_{k}\left(a_{k}^{\dagger}+a_{k}\right) \sigma_{x}+\sum_{k} \omega_{k} a_{k}^{\dagger} a_{k}
$$

where the qubit has an energy splitting of $\Delta$ and coupling strength $g_{k}$ to boson modes of frequency $\omega_{k}$. Since MPSbased methods work particularly well on one-dimensional chain models with short range interaction. Hamiltonian (1) is transformed into a representation of a onedimensional semi-infinite chain with nearest interaction through an orthogonal polynomial mapping(see details in Ref. [37]):

$$
H_{\text {chain }}=\frac{1}{2} \Delta \sigma_{z}+\frac{\sigma_{x}}{2} c_{0}\left(b_{0}+b_{0}^{\dagger}\right)+\sum_{k=0}^{L}\left[\epsilon_{k} b_{k}^{\dagger} b_{k}+t_{k}\left(b_{k}^{\dagger} b_{k+1}+b_{k+1}^{\dagger} b_{k}\right)\right]
$$

where $b_{k}^{\dagger}\left(b_{k}\right)$ are creation(annihilation) operators for transformed new boson modes with $\epsilon_{k}$ describing their frequency and $t_{k}$ representing the internal nearest coupling strength. $c_{0}$ characterizes the effective coupling between the system and new effective environment. $t_{k}, \epsilon_{k}$ and $c_{0}$ are determined by the specific form of the spectral function $J(\omega)=\sum_{k} g_{k}^{2} \delta\left(\omega-\omega_{k}\right)$. The truncation site number $L$ is set to be large enough to ensure convergence.

Instead of using standard matrix product representation with fixed local eigenbasis of boson modes $\left|n_{k}\right\rangle$ (with truncation number $d_{k}$ ), an optimized boson basis $\left|\tilde{n}_{k}\right\rangle$ (with truncation number $d_{O, k} \ll d_{k}$ ) is further employed through an additional isometric map $V_{\tilde{n}_{k}, n_{k}}$, which was introduced in Ref. [38] to study the quantum criticality of the spin-boson model. In the case of a large variance of photon number, this mapping allows significant compression of local boson basis and dramatically enlarges the maximal photon number attainable during dynamical evolution.

The TDVP introduced in Ref. [35] is employed to calculate the evolution here. It is based on the Dirac-Frenkel variational principle by projecting the Schrödinger equation onto the tangent space of the MPS manifold. The obtained optimal equation shares a similar form with the original MPS algorithm based on the Suzuki-Trotter decomposition 39], evolving the system site by site in small time steps $\delta t$. A further improvement was given in Ref. 31] to incorporate the optimized boson basis in the equation of motion. It was proved that TDVP is equivalent to a Lie-Trotter splitting approach [40], where one evolves each integrable part of the Schrödinger equation in the MPS framework. Unlike the Suzuki-Trotter decomposition of the time-evolution operator $U(t)=e^{-i H t}$, errors of TDVP arise only in the integration scheme.

We outline briefly the approach. The MPS algorithm works well on any one-dimensional chain model (2), the optimized boson basis is added to the original MPS network to increase the maximal photon number in the Fock basis. Then TDVP is employed to calculate the evolution. The error of the whole algorithm comes from the following procedures. First, MPS-bond dimension $D$ bounds the maximal entanglement of the state that can be described in current MPS subspace. Second, the truncation numbers, $d_{O, k}$, of the local basis $d_{k}$ and the optimized boson basis restrict the maximal attainable photon numbers and therefore the environmental quantum fluctuations. Finally, time step $\delta t$ introduces an error of integration in the equation of motion. All of these errors can 
be well controlled by setting the parameters adequately large (for $D, d_{k}, d_{O, k}$ ), or small (for $\delta t$ ) to obtain a numerically reliable result. Unless otherwise specified, we set $D=6, d_{k}=40, d_{O, k}=12 \sim 20$ and $\delta t=0.1 \sim 0.4$ for all of the calculations in this work. In addition, to avoid the reflection of the evolution from the end of the chain, we generally choose the size of the chain in the Hamiltonian (2) $L=\frac{2}{3} \omega_{c} T$ ( $\mathrm{T}$ is the simulated time range). All results in the rest of the paper are carefully checked to reach convergence.

In this work, we focus on the power-law spectral function which can be written as:

$$
J(\omega)=\sum_{k} g_{k}^{2} \delta\left(\omega-\omega_{k}\right)=2 \alpha \omega^{s} \omega_{c}^{1-s} \Theta\left(\omega_{c}-\omega\right),
$$

where $\alpha$ is the dimensionless coupling strength, $\omega_{c}$ is the maximal frequency of the environment, and $\Theta(x)$ is the step function. The bath exponent $s$ classifies the reservoir into super-Ohmic $(s>1)$, Ohmic $(s=1)$, and subOhmic $(s<1)$ types respectively. We set $\omega_{c}$ as the energy unit. For the data presented below, we typically choose $\Delta / \omega_{c}=0.1$. All quantities plotted throughout this paper are dimensionless.

\section{SPIN DYNAMICS}

We first study the spin dynamics and compare the result of the TDVP method with previous analytical studies based on a conventional unitary transformation approach proposed in Ref. [14].

The basic idea of the UT approach is to perform a polaronic transformation with a certain shift $\lambda_{k}$ for each boson mode to the rotated spin-boson Hamiltonian,

$$
H^{\prime}=\exp (S) H \exp (-S)
$$

where

$$
S=\sum_{k} \lambda_{k}\left(a_{k}^{\dagger}-a_{k}\right) \sigma_{x}, \lambda_{k}=\frac{g_{k}}{2 \omega_{k}} \xi_{k},
$$

followed by dropping the counter-rotating terms after the transformation. The shift $\lambda_{k}$ are determined variationally by minimizing the expectation value of the higher order terms on the ground state of the transformed Hamiltonian. The transformed Hamiltonian is of the RWA type [14], which thus can be used as a versatile platform to study quantum phase transitions, nonequilibrium dynamics and Zeno effects in the spin-Boson model [15, 16, 41, 42] as straightforwardly as in the original RWA framework.

In the previous UT study [14, the ground state of the transformed Hamiltonian $H^{\prime}$ in the RWA form is easily obtained as $|G S\rangle^{\prime}=|\downarrow\rangle \otimes\left|\{0\}_{k}\right\rangle$. The dynamics can then be straightforwardly studied for an initial state

$$
|\psi(t=0)\rangle^{\prime}=\frac{1}{\sqrt{2}}\left(1+\sigma_{x}\right)|G S\rangle^{\prime}
$$

It takes the following form for the original Hamiltonian (11)

$$
|\psi(t=0)\rangle=\frac{1}{\sqrt{2}}\left(1+\sigma_{x}\right) \exp [-S]|\downarrow\rangle\left|\{0\}_{k}\right\rangle
$$

This initial state is the same as in Eq.(6) of Ref. 41] for the spin-boson Hamiltonian.

The spin dynamics $\left\langle\sigma_{x}^{\prime}(t)\right\rangle$ in the framework of Hamiltonian (3) can be straightforwardly calculated by UT approaches. To show the effectiveness of the UT approach, we also use TDVP to calculate $\left\langle\sigma_{x}^{\prime}(t)\right\rangle$ by the evolution of initial states (5) to Hamiltonian (10). As shown in Fig. 1. both methods give an oscillatory decay for the coherence $\left\langle\sigma_{x}^{\prime}(t)\right\rangle$ and agree well for a long period of time in the weak coupling regime $(\alpha=0.05)$ of the Ohmic reservoir. When the coupling strength increases, the UT results obviously deviate from the TDVP ones. It follows that the UT approach is only suitable in the weak coupling regime, where some analytical results are available. However, as the coupling strength increases, it becomes obvious that the UT approach does not describe the correct dynamics. The significant differences between RWA and non-RWA in the strong coupling regime have been reported in Ref. ( 23]). The newly developed TDVP approach would be a better candidate. In the next section, we generalize the TDVP approach to study the QZE and QAZE.

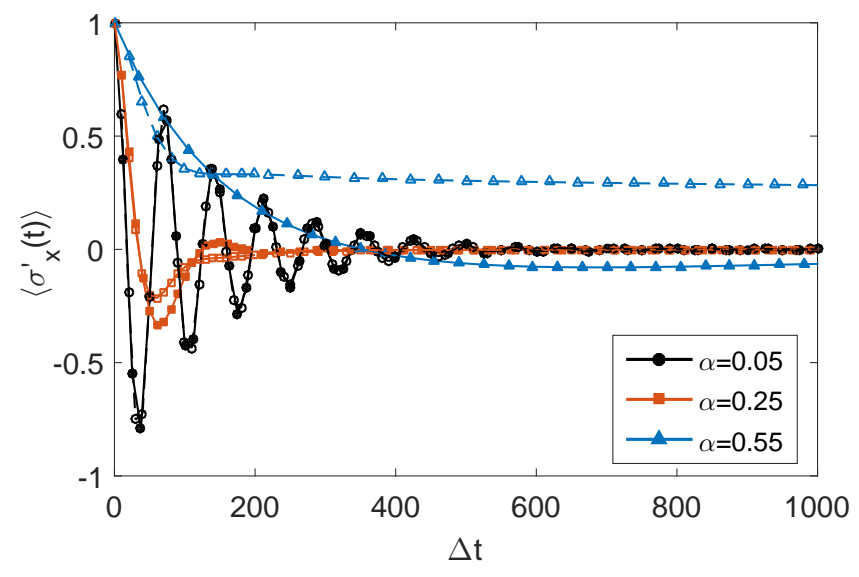

FIG. 1: (Color online) Evolution of the coherence $\left\langle\sigma_{x}^{\prime}(t)\right\rangle$ for the Hamiltonian (3) from the initial state Eq. (4). Lines with solid symbols denote the results from TDVP while those with open symbols are results of UT. Both methods show good agreement only at weak coupling $(\alpha=0.05)$.

\section{QZE AND QAZE TRANSITION IN OPEN QUANTUM SYSTEM}

With this efficient numerical technique, we turn to the QZE and QAZE in open quantum system described by Hamiltonian (10). To study the QZE-QAZE transition we focus on the survival probability $P_{s}(t=n \tau)$ [1], defined as the probability of finding initial state after $N$ 
successive measurements with equal time interval $\tau$ (zero temperature):

$$
P_{s}(t=n \tau)=|\langle\psi(0)|\exp (-i H \tau)| \psi(0)\rangle|^{2 n}
$$

If the time interval $\tau$ is short enough, we can further simplify it:

$$
P_{s}(t=n \tau) \approx\left[1-\left(\left\langle H^{2}\right\rangle-\langle H\rangle^{2}\right) \tau^{2}\right]^{n} \approx \exp (-\gamma(\tau) t)
$$

where $\gamma(\tau)$ is the effective decay rate induced by measurements. The exponential form of $P_{s}(\tau)$ in Eq. (6) is valid under certain conditions. First, $\tau$ should be shorter than the typical time scale of the evolution without measurement. This can be fulfilled by limiting the time scale in the calculation to be shorter than the time scale of the bare Rabi oscillation, namely $t \leq \pi / \Delta$. Moreover, the correlation between qubit and environment should be weak so that the environment experiences no significant evolution during the interval of repetitive measurements leading to the assumption that the whole system (qubit plus its environment) collapses to its initial state after each measurement. This assumption, although commonly employed in previous studies, becomes invalid when the system and its environment is strongly correlated due to the strong coupling between them. This system-environment correlation effects will affect the properties of QZE-QAZE transition. To compare with previous studies, we present our results also under this assumption first, then discuss the Zeno effect beyond this assumption, i. e. if only the qubit is measured.

In the study of Zeno effects, two initial states are widely used in the literature. One is the product state of the photon vacuum and the atomic excited state [1, 18],

$$
\left|\psi_{1}(t=0)\right\rangle=|\uparrow\rangle \otimes\left|\{0\}_{k}\right\rangle
$$

which we denote as the bare bath initial state. In the UT approach [15, 16], the initial state is usually chosen as

$$
\left|\psi_{2}(t=0)\right\rangle=\exp [-S]\left(|\uparrow\rangle \otimes\left|\{0\}_{k}\right\rangle\right) .
$$

Note that $|\uparrow\rangle \otimes\left|\{0\}_{k}\right\rangle$ is obtained by flipping the spin of the ground state of the transformed Hamiltonian (3) in the RWA form, and thus we call state (8) the physical bath initial state. It is an entangled state of the system and the environment, and just corresponds to the state of the measured system prepared in an eigenstate of the measured observable. On the Bloch sphere form of qubit, one can write it as

$$
\left|\psi_{2}(t=0)\right\rangle=\cosh (B)\left|\{0\}_{k}\right\rangle|\uparrow\rangle-\sinh (B)\left|\{0\}_{k}\right\rangle|\downarrow\rangle,
$$

where $B=\sum_{k} \lambda_{k}\left(a_{k}^{\dagger}-a_{k}\right)$. Both initial states (7) and (8) will be employed to study the Zeno effects in this paper.

\section{A. Measuring the whole system}

In this section, we measure the whole system by projecting the total wave function onto the initial state. It means that we do not consider the system-environment correlation effects in the measurement. Two initial states are considered:

Physical bath initial state: The Zeno effect is easily studied within the UT approach [14] for the physical bath initial state (8). So we perform the TDVP study with the same initial state. The comparison between the TDVP and UT method is presented in Fig. 2 for bath exponent $s=1$ and 0.75 . As expected, the two methods agree well in the weak coupling regime [e.g. $\alpha=0.05, s=1$ and $\alpha=0.025, s=0.75]$, since the counter-rotating terms play only a little role in this regime, and the partial consideration of their effects in the UT study is a very good approximation. It should be noted that the decay rate increases monotonically with the increase of the time interval of measurements in the weak coupling regime. This monotonicity can be regarded as a new definition of the Zeno effect [43], since the evolution of the system is slowed down by frequent measurement. The QAZE can be defined in an analogous manner as the nonmonotonicity of $\gamma(\tau)$, i.e. the decay rate increases first and then decreases again with the measurement time interval. This definition retains the core physical content of QZE and QAZE, namely that frequent measurement either slows down or accelerates the evolution. Moreover, it avoids calculating the decay rate for the infinitely long measurement interval which can be hardly obtained accurately by numerical methods if no analytical solutions are available, such as in the present model. Throughout this paper, we define QZE and QAZE by the functional behavior of $\gamma(\tau)$.

When the coupling strength increases, the UT result obviously deviates from the numerically exact TDVP method. Interestingly, a non-monotonic behavior is indeed found for strong coupling in the TDVP curves, which is never exhibited by the UT curves indicated by dashed lines in Fig. 2, The TDVP method predicts a transition from QZE to QAZE for $\alpha \geq 0.6$ around $\Delta \tau=0.2$ and Ohmic bath. Note that UT fails to capture this feature and only shows the QZE in all coupling regime 14. It was demonstrated in Ref. 32] that the dynamics in the strong coupling regime is considerably affected by the multi-photon process described by higher order non-RWA terms while the UT method neglects most of these higher-order terms. Note also that the significant differences between RWA and non-RWA in the strong coupling regime was reported in Ref. [28]. It is just these higher-order non-RWA terms that are responsible for the QZE-QAZE transition at the strong coupling.

Bare bath initial state: Starting the evolution from the widely used state (7), the QZE-QAZE transition is always observed both for the Ohmic and sub-Ohmic baths, as shown by solid lines in Fig. 3. for all coupling 

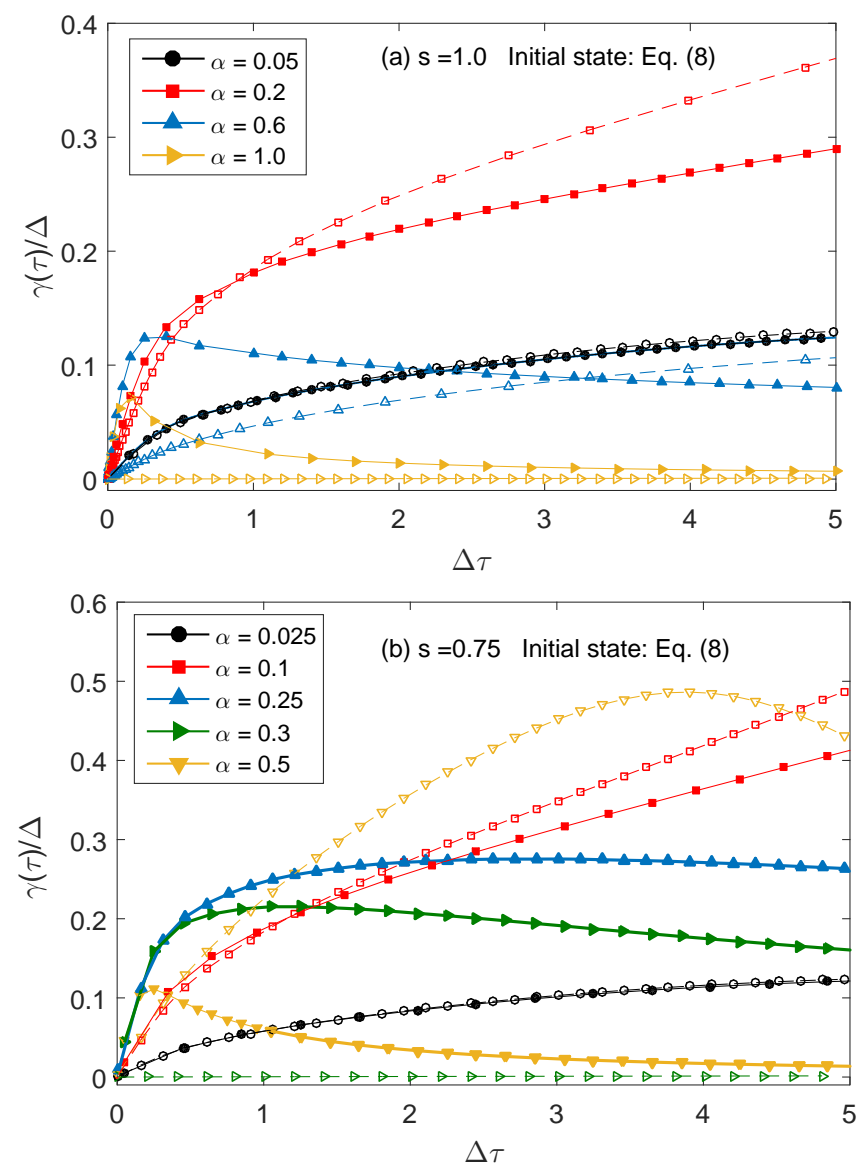

FIG. 2: (Color online) Zeno decay rate from physical bath initial states obtained by TDVP (solid lines with filled symbols) and UT (dashed lines with open symbols) methods for (a) $s=1$ and (b) $s=0.75$. Specifically, thick solid lines denote the TDVP results for coupling strengths above the critical point in the ground state.

strengths. The previous RWA results, only applicable at extremely weak coupling, also support these findings.

We conclude that the presence of the QZE-QAZE transition is highly dependent on the choice of the initial state. The UT study for the physical bath initial state can only be applied for weak coupling. In the present exact study, we find that this suppression of QAZE is an artefact of the UT approximation and the QAZE reappears when the coupling strength increases. On the other hand, for the often-used bare bath initial state, the QZEQAZE transition always obtains at arbitrary coupling.

In the spin-boson model, it is well known that the second-order quantum phase transition from delocalization to localization occurs for sub-Ohmic bath [44]. While for the Ohmic bath, the delocalized-localized quantum phase transition is of Kosterlitz-Thouless type [45]. For the parameter used in this paper, the critical coupling strengths are around $\alpha_{c}=1$ for $s=1$ and 0.295 for $s=0.75$ [44]. From Fig. 2 we observe that the onset of the QZE-QAZE transition takes place below the corre-
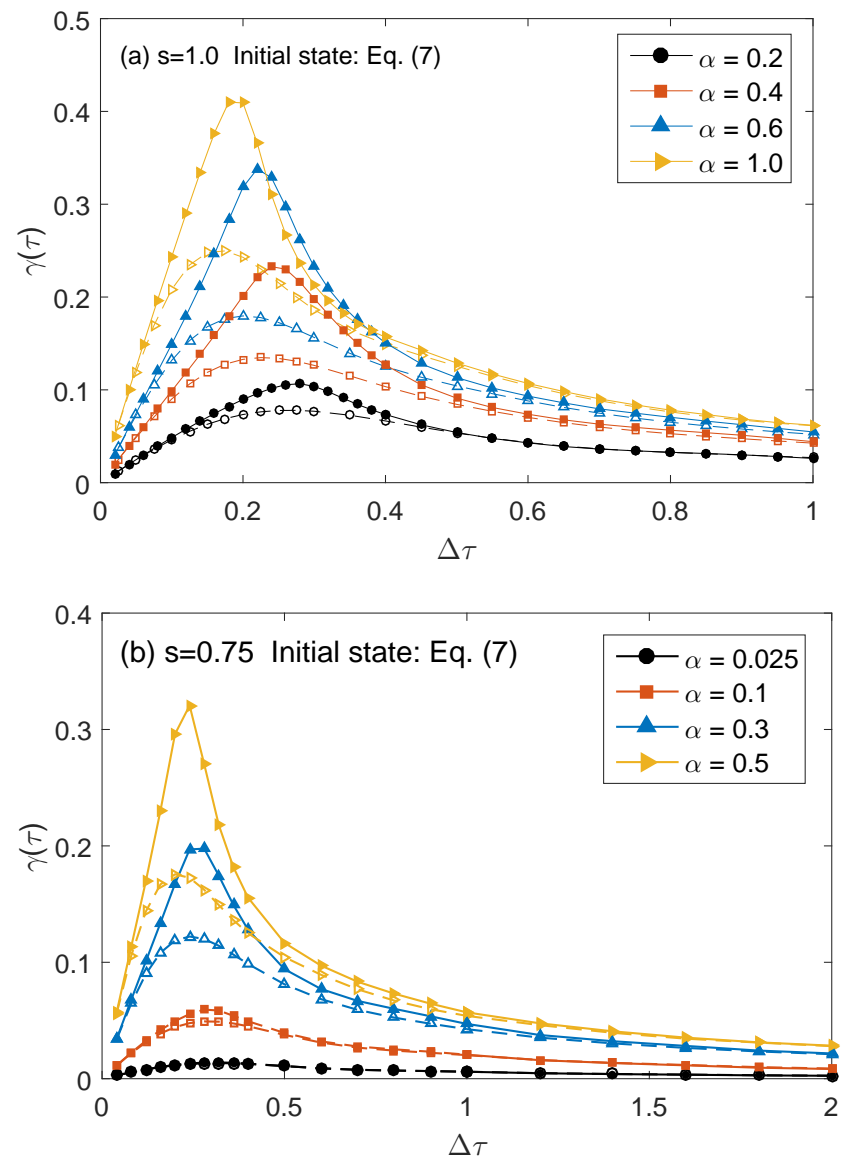

FIG. 3: (Color online) The decay rate from the bare bath initial states obtained by TDVP for (a) $s=1$ and (b) $s=0.75$. Results with the previous measurement scheme are indicated by lines with open symbols and those with the new measurement scheme by lines with filled symbols.

sponding critical points for both Ohmic and sub-Ohmic baths, revealing that the presence of the localized, or delocalized phase does not affect the QZE-QAZE transition. In the study of Zeno effect, the incoherent decay of excited states towards the ground state is highly nontrivial. The reason that these ground state delocalization to localization transitions do not affect the the QZE-QAZE transition is worthy of further study. The preliminary understanding may be the following. The QZE and QAZE are very sensitive to the earlier dynamics, the state evolving at the earlier stage is far away from the final groundstate. So the ground-state phase has no dominant effect on the earlier state. Even after the long time evolution, the ground state have no essential effect. Above the critical point, the two states of the qubit are present with equal probability, so there is no influence at all. Below the critical point, a doubly degenerate localized phase is formed, the preferred spin state only depends on the initial state, which is however independent of the coupling strength. 


\section{B. Measuring the qubit only}

In the previous section, the measurement resets both the system and environment to the total initial state. As explained in the Introduction, this assumption is reasonable only if the coupling strength is weak and the measurement is performed with high frequency. In the general case, each measurement projects only the system (here the qubit) onto its initial state, while the environment still evolves and never falls back to its initial state upon measurement. Here we describe a more general scheme suited to this case for the widely used bare bath initial state (7).

After unitary evolution in a period of time $t$, the state can be written as

$$
|\psi(t)\rangle=C_{1}|\uparrow\rangle \otimes\left|\phi_{\text {env }}^{1}\right\rangle+C_{2}|\downarrow\rangle \otimes\left|\phi_{\text {env }}^{2}\right\rangle
$$

where $\left|\phi_{\mathrm{env}}^{i,=1,2}\right\rangle$ is the environmental state of photons. Measuring the qubit only (and projecting it by assumption onto its initial spin-up state) will lead to the new initial state:

$$
|\psi(t)\rangle_{\text {new }}=|\uparrow\rangle \otimes\left|\phi_{\text {env }}^{1}\right\rangle .
$$

Note that the environmental part is always different from the initial photon vacuum after the measurement due to the back-reaction of the system to the environment, no matter how close they may be.

The decay rates for both the previous and the present measurement scheme are shown in Fig. 3 for exponents $s=1$ and $s=0.75$. It is interesting that the decay rates differ markedly only for intermediate time intervals. The qualitative nature of the QZE-QAZE transition remains unchanged for all coupling strengths.

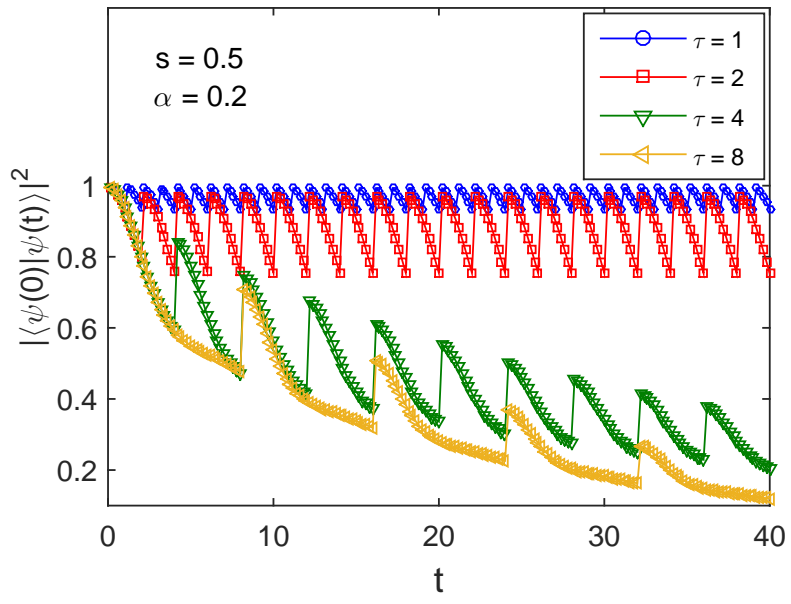

FIG. 4: (Color online) Evolution of the fidelity between current state and initial state for different measurement time intervals in a sub-Ohmic bath. The initial state is chosen as the bare bath initial state(7).

To explain the above findings qualitatively, we calculate the time dependence of the fidelity between the current state and the initial state in the new measurement scheme for the sub-Ohmic $(s=0.5)$ spin-boson model at strong coupling $(\alpha=0.2)$. It is clearly seen from Fig. 4 that the fidelity depends on the time interval $\tau$.

For fast frequent measurements like $\tau=1 \sim 2$, the time interval is so short that the environment cannot evolve far from its initial state, although the correlation between the system and environment is strong, and the fidelity almost remains the same (unity) after each measurement. As shown in Fig. 5 (a), the bath has only less than one photonic excitation for the whole range of frequencies during the measurement interval. The evolution of the environment exhibits an obvious periodicity, indicating that the system plus its environment return to the initial state after each measurement. In this case, the measurement scheme of the previous section is not qualitatively different from the present new one. It follows that the previous assumption still works well for fast measurements.

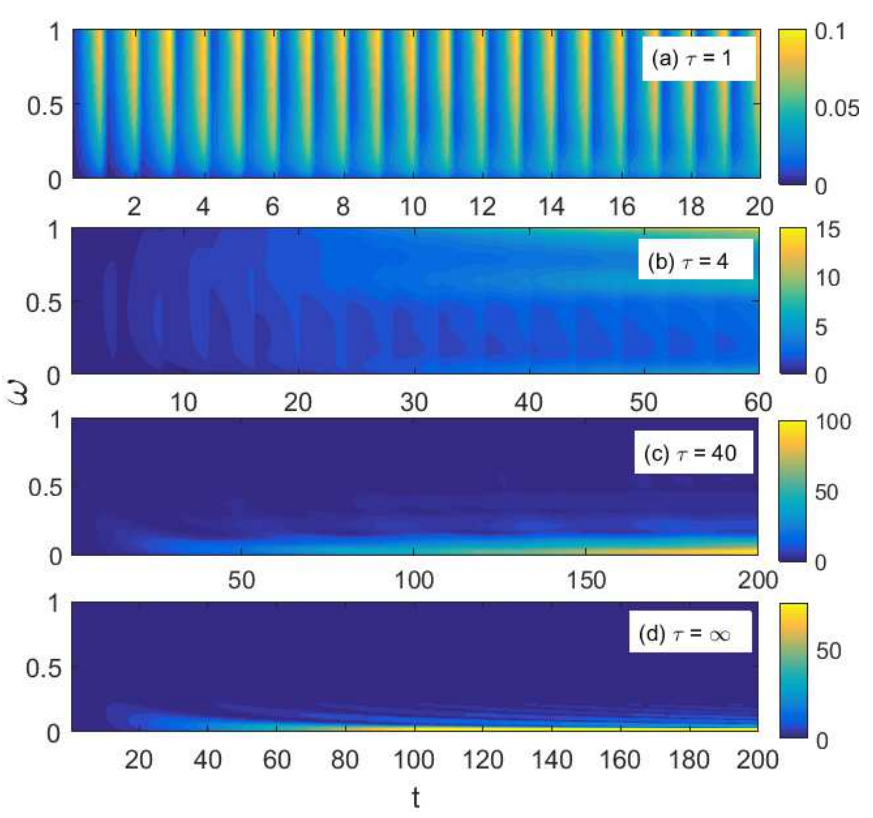

FIG. 5: (Color online) Evolution of numbers of photons with different mode frequencies in the environment for 4 typical measurement time intervals when the qubit is measured only. The initial state is chosen as the bare bath initial state (7). $s=0.5, \alpha=0.2$.

However, for slow measurements, $\tau=4 \sim 8$, the fidelity dramatically deviates from unity already after the second measurement, manifesting the breakdown of the previous assumption. As demonstrated in Fig. 5(b), the net excitations of the bath photonic numbers are accumulated through successive measurement, especially for the high frequency part of the environment. In this case, each measurement generates a new environmental state which obvious evolves and deviates from its initial form due to the strong correlation between the system and the environment. This new initial state carries information from the previous evolution-measurement cycle into the 
next one, inducing a non-Markovian effect to the whole repetitive measurement process. The survival probability of each measurement turns out to be time-dependent and finally enhances the decay rate.

When the measurement interval is large enough, exceeding the relaxation time (or memory time of the bath), the qubit approximately relaxes to a quasi-steady state which is independent from the initial state of the bath. It is exhibited in Fig. 5 (c) for a relatively long time $(\tau=40)$ that the evolution of the bath during the measurement interval shows a gradually excitations of the low frequency photons, similar to the one without measurements shown in Fig. 5(d). In this extremely slow measurements, the decay rate remains unchanged with time, just demonstrated in the curves for large $\tau$ in Fig. 3 .

\section{CONCLUSION}

In summary, we study the Zeno effect in the open quantum system of a qubit interacting with a bosonic bath by using a numerically exact method based on the MPS. We find that the QZE-QAZE transition is highly dependent on the initial state if measuring both spin (qubit) and environment, which has been usually adopted in the literature. For the bare bath initial state, the QZE-QAZE transition happens always, independent of the coupling strength. For the physical bath initial state, the QZEQAZE transition is absent at weak coupling but reappears at strong coupling. Thus the previous result of the UT-based study showing a suppression of QAZE [15, 17] has to be modified.

Furthermore, we consider a more realistic measurement process which only projects the qubit to its ini- tial state while the environment continues to change. In the new measurement scheme, we find that QZE-QAZE transition happens always, independent of the coupling strength, for the bare initial state. In addition, measuring the qubit only leads to a faster decay rate for intermediate measurement time intervals.

All the above statements holds for both Ohmic and sub-Ohmic baths. We did not find any dependence of the above observations on the coherent-incoherent crossover and/or the delocalized-localized phase transition, indicating that these effects qualitatively play no role for the QZE to QAZE transition.

Note added: Recently, we became aware of a paper [46] where the quantum Zeno and anti-Zeno effects were studied in a spin-boson model with Lorentzian-like spectrum where the exact hierarchical equations-of-motion approach can be applied. For the physical bath initial state, multiple Zeno-to-anti-Zeno crossovers were observed, possibly due to the oscillation of the population dynamics in the Lorentzian bath as shown in Fig. 1 of Ref. [46]. The Lorentzian bath is usually used to describe a bad cavity, so the population dynamics is similar to the case of single (lossy) cavity, like the quantum Rabi model. In contrast, for Ohmic and sub-Ohmic baths we find here that the QZE-QAZE transition usually occurs only once.

ACKNOWLEDGEMENTS This work was supported by th National Natural Science Foundation of China under Grants No. 11674285 and No. 11474256. We thank D. Braak for critically reading this manuscript and many helpful suggestions. We also acknowledge useful discussions with C. Wang.

* Email:qhchen@zju.edu.cn
[1] A. G. Kofman and G. Kurizki, Nature (London) 405, 546 (2000); Phys. Rev. A 54, R3750 (1996).

[2] P. Facchi, H. Nakazato, and S. Pascazio, Phys. Rev. Lett. 86, 2699 (2001).

[3] A. Barenco, A. Berthiaume, D. Deutsch, A. Ekert, R. Jozsa, and C. Macchiavello, SIAM Journal on Computing 26, 1541 (1997)..

[4] A. Beige, D. Braun, B. Tregenna, and P. L. Knight, Phys. Rev. Lett. 85, 1762 (2000).

[5] N. Erez, G. Gordon, M. Nest, and G. Kurizki, Nature 452, 724 (2008).

[6] W. M. Itano, D. J. Heinzen, J. Bollinger, and D. Wineland, Phys. Rev. A 41, 2295 (1990)

[7] E. W. Streed, J. Mun, M. Boyd, G. K. Campbell, P. Medley, W. Ketterle, and D. E. Pritchard, Phys. Rev. Lett. 97, 260402 (2006).

[8] P. W. Chen, D. B. Tsai, and P. Bennett, Phys. Rev. B 81, 115307 (2010)

[9] A. Barone, G. Kurizki, and A. Kofman, Phys. Rev. Lett. 92, 200403 (2004)

[10] F. Helmer, M. Mariantoni, E. Solano, and F. Marquardt, Phys. Rev. A 79, 052115 (2009).
[11] J. Bourassa, J. M. Gambetta, A. A. Abdumalikov Jr, O. Astafiev, Y. Nakamura, and A. Blais, Phys. Rev. A 80, 032109 (2009).

[12] A. A. Abdumalikov Jr, O. Astafiev, Y. Nakamura, Y. A. Pashkin, and J. Tsai, Phys. Rev. B 78, 180502 (2008).

[13] P. Forn-Díaz et al., Nature Physics 13, 39 (2017).

[14] Z. G. Lv and H. Zheng, Phys. Rev. B 75, 054302(2007).

[15] H. Zheng, S. Zhu, and M. Zubairy, Phys. Rev. Lett. 101, 200404 (2008).

[16] X. Cao, J. You, H. Zheng, A. Kofman, and F. Nori, Phys. Rev. A 82, 022119 (2010).

[17] Q. Ai, Y. Li, H. Zheng, and C. P. Sun, Phys. Rev. A 81, 042116 (2010)

[18] P. Facchi and S. Pascazio, Phys. Lett. A 241, 139 (1998).

[19] Y. Tanimura and R. K. Kubo, J. Phys. Soc. Japan 58, 101 (1989); A. G. Dijkstra and Y. Tanimura, Phys. Rev. Lett. 104, 250401 (2010).

[20] J. S. Shao, J. Chem. Phys. 120, 5053 (2004); Y. A. Yan, F. Yang, Y. Liu and J. S. Shao, Chem. Phys. Lett. 395, 216 (2004).

[21] R. X. Xu and Y. J. Yan, Phys. Rev. E 75, 031107 (2007).

[22] H. B. Wang and M. Thoss, New Journal of Physics 10, 
115005 (2008).

[23] Prior, J., Chin, A. W., Huelga, S. F., Plenio, M. B, Phys. Rev. Lett. , 105, 050404 (2010).

[24] C. K. Lee, J. M. Moix, and J. Cao, J. Chem. Phys. 136, 204120 (2012); C. K. Lee, J. Cao, and J. B. Gong Phys. Rev. E 86, 021109 (2012).

[25] N. Wu, L. W. Duan, X. Li, and Y. Zhao, J. Chem. Phys. 138, 084111 (2013).

[26] J. Ma, Z. Sun, X. Wang, and F. Nori, Phys. Rev. A 85, 062323 (2012).

[27] C. Wang and Q. H. Chen, New Journal of Physics 15, 103020 (2013); L. W. Duan, H. Wang, Q. H. Chen, and Y. Zhao,, J. Chem. Phys. 139, 044115 (2013).

[28] Prior, J., de Vega, I., Chin, A. W., Huelga, S. F., Plenio, M. B. Phys. Rev. A 87, 013428 (2013)

[29] Chin, A. W., Prior, J., Rosenbach, R., Caycedo-Soler, F., Huelga, S. F., Plenio, M. B. Nature Physics 9, 113 (2013)

[30] D. Kast and J. Ankerhold, Phys. Rev. Lett. 110, 010402 (2013).

[31] F. A. Y. N. Schröder, A. W. Chin, Phys. Rev. B 93, 075105 (2016).

[32] Z. Blunden-Codd et al., Phys. Rev. B 95, 085104(2017).

[33] G. Gordon, D. B. Rao, and G. Kurizki, New Journal of Physics 12, 053033 (2010).

[34] A. Z. Chaudhry and J. Gong, Phys. Rev. A 90, 012101 (2014).

[35] J. Haegeman et al., Phys. Rev. B 94, 165116 (2016).
[36] Shu He, Ph.D Thesis (Zhejiang University) submitted in December 2016, Chapter 5.

[37] A. W. Chin, A. Rivas, S. F. Huelga, and M. B. Plenio, Journal of Mathematical Physics 51, 092109 (2010).

[38] C. Guo, A. Weichselbaum, J. von Delft, and M. Vojta, Phys. Rev. Lett. 108, 160401 (2012).

[39] M. Suzuki, Physics Letters A 146, 319 (1990).

[40] M. Suzuki, Communications in Mathematical Physics 51, 183 (1976).

[41] Q.-J. Tong, J.-H. An, H.-G. Luo, and C. Oh, Phys. Rev. B 84, 174301 (2011).

[42] H. Zheng and Z. G. Lu, J. Chem. Phys. 138, 174117 (2013); H. Zheng, Z. G. Lü, and Y. Zhao, Phys. Rev. E 91, 062115 (2015).

[43] I. Lizuain, J. Casanova, J. J. Garcia-Ripoll, J. Muga, and E. Solano, Phys. Rev. A 81, 062131 (2010).

[44] A. Winter, H. Rieger, M. Vojta, and R. Bulla, Phys. Rev. Lett. 102, 030601 (2009); A. Alvermann and H. Fehske, Phys. Rev. Lett. 102, 150601 (2009); Y. Y. Zhang, Q. H. Chen and K. L. Wang, Phys. Rev. B 81, 121105(R) (2010); A. W. Chin, J. Prior, S. F. Huelga and M. B. Plenio, Phys. Rev. Lett. 107, 160601 (2011).

[45] A. J. Leggett et al., Rev. Mod. Phys. 59, 1(1987); U. Weiss, Quantum Dissipative Systems (World Scientific, Singapore, 1993).

[46] W. Wu and H. -Q. Lin, Phys. Rev. A 95, 042132 (2017). 